\title{
Datation d'un vestige humain provenant de la Rochette (Saint Léon-sur-Vézère, Dordogne) par la méthode du carbone 14 en spectrométrie de masse
}

The AMS radiocarbon dating of human remains from La Rochette (Saint-Léonsur-Vézère, Dordogne)

Die AMS 14C Datierung der Menschenreste von La Rochette (Saint-Léon-sur-

Vézère, Dordogne)

Jörg Orschiedt

\section{OpenEdition \\ Journals}

Édition électronique

URL : http://journals.openedition.org/paleo/1491

DOI : $10.4000 /$ paleo.1491

ISSN : $2101-0420$

Éditeur

SAMRA

Édition imprimée

Date de publication : 1 décembre 2002

Pagination : $239-240$

ISSN : 1145-3370

Référence électronique

Jörg Orschiedt, « Datation d'un vestige humain provenant de la Rochette (Saint Léon-sur-Vézère, Dordogne) par la méthode du carbone 14 en spectrométrie de masse », PALEO [En ligne], 14 | 2002, mis en ligne le 17 août 2010, consulté le 27 juillet 2020. URL : http://journals.openedition.org/paleo/ 1491 ; DOI : https://doi.org/10.4000/paleo.1491

Ce document a été généré automatiquement le 27 juillet 2020.

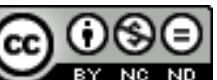

PALEO est mis à disposition selon les termes de la licence Creative Commons Attribution - Pas d'Utilisation Commerciale - Pas de Modification 4.0 International. 


\section{Datation d'un vestige humain provenant de la Rochette (Saint Léon-sur-Vézère, Dordogne) par la méthode du carbone 14 en spectrométrie de masse}

The AMS radiocarbon dating of human remains from La Rochette (Saint-Léonsur-Vézère, Dordogne)

Die AMS 14C Datierung der Menschenreste von La Rochette (Saint-Léon-surVézère, Dordogne)

Jörg Orschiedt

Le site peu connu de l'abri de La Rochette (vallée de la Vézère) a été désigné comme "ruine archéologique" (Delporte 1962, p. 1). Les fouilles de O. Hauser en 1910 et surtout celles de H. Delporte en 1961 et 1962 ont permis de relever une séquence stratigraphique importante. Pendant les travaux de O. Hauser (Hauser 1911), la plupart des couches archéologiques ont été fouillées (abri principal, secteur B). Les fouilles de H. Delporte avaient lieu dans le secteur A, une carrière du XIX ${ }^{\text {ème }}$ siècle, à l'est de l'abri principal et à l'ouest au secteur C (Delporte 1962, fig. 1). Si les fouilles de O. Hauser n'ont fourni qu'une séquence stratigraphique simplifiée et peu juste, les recherches de H. Delporte ont, heureusement, livré des résultats beaucoup plus précis et détaillés.

- La stratigraphie de La Rochette d'après H. Delporte (1962) est la suivante :

- Gravettien (Périgordien supérieur, Faciès Noailles) - couche 2 (secteur A),

- Aurignacien - couches 3, 4, 5,

- Châtelperronien - couche 6,

- Moustérien terminal - couche 7,

- Moustérien tradition acheuléenne - couches 8-9,

- Moustérien type charentien - couche 10. 

milieu des niveaux aurignaciens 3 à 5 . Près de ces vestiges ont été également trouvés plusieurs dents de chevaux, percées, qui, selon 0 . Hauser, pourraient correspondre à d'éventuels bijoux portés au niveau des mains (Hauser 1927). Les fossiles humains sont représentés par des fragments d'un humérus droit et gauche, d'une ulna et d'un radius droit, d'un fémur droit presque complet ainsi qu'un autre fragment du côté gauche. A cela s'ajoutent onze dents, qui peuvent être attribuées à trois individus d'âges différents. Il s'agit d'un adulte, d'un adolescent et d'un enfant. L'analyse anthropologique a été publiée par H. Klaatsch et W. Lustig (1914) ainsi qu'une analyse odontologique par F. W. Elsner (1914). Actuellement, les vestiges de La Rochette sont inventoriés comme La Rochette 1 et 2 (os long) et La Rochette 3 et 5 (dents) dans le "Catalogue of Fossil Hominids" (Oakley et al. 1971, 172). Mais, à cause de doutes importants concernant les méthodes de travail de 0 . Hauser, la position chronologique des vestiges humains de La Rochette a été sérieusement mise en doute (May 1986, p. 55 ; Binant 1991, p. 102). vestiges humains du même site faisant partie des collections du Musée National de Préhistoire aux Eyzies de Tayac. Elle donne un âge de $1.610 \pm 80$ B.P. (GifA-95455) et ainsi un âge calibré de 253 à 612 A.D. (Gambier et al., 2000). ("whereabouts unknown") (Oakley et al. 1971, p. 172), un ré-examen de ces derniers n'avait pu avoir lieu. Depuis, ces fossiles ont été retrouvés dans la collection ostéologique de l'Université de Tübingen (Allemagne). Ils sont inventoriés sous le numéro 7074. Nous avons donc décidé de réaliser de nouvelles datations absolues sur ces derniers. Une première date, par la méthode ${ }^{14} \mathrm{C}$ SMA, a été obtenue sur un fragment d'ulna droite ${ }^{1}$. La datation donne un âge de $23.630 \pm 130$ B.P. (OxA-11053). Ainsi ce vestige de La Rochette remonterait au Gravettien supérieur.

5 En fonction de ce résultat et de celui obtenu précédemment (Gambier et al., 2000), il nous semble donc important qu'une nouvelle recherche exhaustive, impliquant toutes les personnes qui travaillent sur ces fossiles, soit entreprise sur la totalité des restes humains de La Rochette (ainsi que sur la parure censée provenir du même niveau). Naturellement, ce travail doit s'accompagner d'une analyse aussi précise que possible des archives disponibles sur ce gisement.

\section{BIBLIOGRAPHIE}

BINANT P. 1991 - Les sépultures du Paléolithique. Archéologie Aujourd'hui. Paris : Editions Errance $108 \mathrm{p}$.

DELPORTE H. 1962 - Le gisement paléolithique de la Rochette, commune de Saint-Léon-surVézère, Dordogne. Gallia Préhistoire, V, p. 1-22.

ELSNER F.W. 1914 - Die Zähne von La Rochette. Archiv für Anthropologie 41, p. 127-129. 
GAMBIER D., VALLADAS H., TISNERAT-LABORDE N,. ARNOLD M. et BRESSON F. 2000 - Datation de vestiges humains présumés du Paléolithique supérieur par la méthode du carbone 14 en spectrométrie de masse par accelerateur. Paléo 12, p. 201-212.

HAUSER O. 1911 - Le Périgord préhistorique. Périgueux : Réjou impr. 22 p.

HAUSER O. 1927 - Der Erde Eiszeit und Sintflut. Ihre Menschen, Tiere und Pflanzen. Berlin: Verlag Georg Stilke $370 \mathrm{p}$.

KLAATSCH H. \& LUSTIG W. 1914 - Morphologie der paläolithischen Skelettreste des mittleren Aurignacien der Grotte von La Rochette, Dordogne. Archiv für Anthropologie 41, p. 81-126.

MAY F. 1986 - Les Sépultures Préhistoriques. Étude Critique. Paris : Éditions du C.N.R.S. 264 p.

OAKLEY K.P., CAMPBELL B.G., MOLLESON T.I. 1971 - Catalogue of Fossil Hominids. Part II: Europe. London: Trustees of the British Museum (Natural History) $379 \mathrm{p}$.

\section{NOTES}

1. Le financement de la datation a été généreusement rendu possible par le Musée de Néanderthal à Mettmann.

\section{RÉSUMÉS}

Les vestiges humains découverts par O. Hauser lors de ses fouilles en 1910 du site de La Rochette ont été attribués par ce dernier à l'Aurignacien. Il a été possible de faire une attribution chronologique exacte d'une de ces pièces (datation absolue SMA), présente dans les collections de l'Université de Tübingen en Allemagne. Elle est maintenant rapportée au Gravettien supérieur.

The human remains found during the excavations by Otto Hauser in 1910 in the Middle and Upper Paleolithic site of La Rochette were originally dated to the Aurignacien by stratigraphical observations of the excavator. The exact chronological position of the human remains was estimated by direct accelerator dating. The remains housed at the Osteological Collection at Tübingen, Germany are now attributed to the late Gravettian.

Die menschlichen Skelettreste, die während der Grabungen von Otto Hauser im Jahre 1910 in der mittel - und jungpaläolithischen Fundstelle von La Rochette entdeckt wurden, wurden vom Ausgräber aufgrund stratigrafischer Beobachtungen dem Aurignacien zugewiesen. Durch eine AMS Beschleunigerdatierung konnte die exakte chronologische Zuordnung der in der Osteologischen Sammlung in Tübingen inventarisierten menschlichen Skelettreste festgestellt werden. Demnach muß der Fund von La Rochette in das späte Gravettien datiert werden. 
INDEX

Mots-clés : Paléolithique supérieur, France, Europe, datation radiocarbone, SMA, Gravettien, vestiges humains, sépulture

Schlüsselwörter : Jungpaläolithikum, Frankreich, Europa, Radiokarbondatierung, AMS, Gravettien, menschliche Skelettreste, Bestattung

Keywords : Upper Paleolithic, France, Europe, Radiocarbon dates, AMS, Gravettian, Human remains, burial

\section{AUTEUR}

JÖRG ORSCHIEDT

Universität Hamburg, Archäologisches Institut, Abteilung I: Vor- und Frühgeschichtliche Archäologie, Johnsallee 35, 20148 Hamburg - e-mail : orschiedt@uni-hamburg.de 